\title{
Auditory cortex mediates the perceptual effects of acoustic temporal expectation
}

\author{
Santiago Jaramillo and Anthony M. Zador \\ Cold Spring Harbor Laboratory, 1 Bungtown Road, Cold Spring Harbor, New York 11724, USA
}

\begin{abstract}
When events occur at predictable instants, anticipation improves performance. Knowledge of event timing modulates motor circuits, improving response speed. By contrast, the neuronal mechanisms underlying changes in sensory perception due to expectation are not well understood. We have developed a novel behavioral paradigm for rats in which we manipulated expectations about sound timing. Valid expectations improved both the speed and the accuracy of subjects' performance, indicating not only improved motor preparedness but also enhanced perception. Single neuron recordings in primary auditory cortex revealed enhanced representation of sounds during periods of heightened expectation. Furthermore, we found that activity in auditory cortex was causally linked to the performance of the task, and that changes in the neuronal representation of sounds predicted performance on a trial-by-trial basis. Our results indicate that changes in neuronal representation as early as primary sensory cortex mediate the perceptual advantage conferred by temporal expectation.
\end{abstract}

Attending to moments in time is a powerful cognitive mechanism for exploiting temporal structure in behaviors such as hunting moving prey or playing music in an ensemble. Anticipation of an event can influence the speed of behavioral response as well as our perception of such an event ${ }^{1}$, but how these improvements in perception arise from changes in neuronal activity remains largely unknown. However, it is clear that organisms can take advantage of regularities in the environment to form expectations and predictions which can be used to enhance performance.

The auditory cortex is sensitive to many forms of acoustic regularity ${ }^{2}$. For example, cortical neurons respond more strongly to rarely presented sounds embedded within a regular sequence, even when these sounds are not required to perform a task ${ }^{3}$. In addition, changes in expectation about the frequency of a task-relevant sound modulate the activity of single neurons in auditory cortex ${ }^{4}$. However, although temporal structure is central to the organization of sounds, our understanding of the effects of temporal expectation in the auditory system is largely limited to studies in humans ${ }^{1,5}$ in which it is difficult to study the underlying neuronal mechanisms. In particular, we know little about whether and how auditory temporal expectation changes neuronal representations in the auditory cortex.

Users may view, print, copy, download and text and data- mine the content in such documents, for the purposes of academic research, subject always to the full Conditions of use: http://www.nature.com/authors/editorial_policies/license.html\#terms

AUTHOR CONTRIBUTION

S.J. and A.M.Z. designed the project and wrote the manuscript. S.J. collected the data and performed the analyses. 
Improved performance from temporal expectation could arise from enhanced motor preparedness, but could also be due to a perceptual enhancement. We have developed a novel behavioral paradigm in rats to study the neuronal mechanisms underlying the perceptual consequences of temporal expectation. Here, for the first time in an animal model, we characterize the effect of expectation about the timing of sounds on both the speed and accuracy of performance. This paradigm allows us to assess changes in perception due to expectation, establish a causal link between neuronal activity and perception, and quantify changes in sensory representation due to temporal expectation. We show for the first time that temporal expectation modulates activity in primary sensory cortex, and that the influence of expectation on neuronal activity in sensory cortex is directly related to the observed improvements in performance. Our results suggest that improvements in auditory perception due to valid temporal expectation arise from changes in sensory representations even at the first stages of cortical processing.

\section{RESULTS}

Our study consists of three sets of experiments designed to evaluate the effects of temporal expectation on sensory areas underlying the perception of sounds. First, we performed behavioral experiments in which we quantified the effect of temporal expectation on the perception of sounds by measuring the reaction time and accuracy of rats performing a detection/discrimination task. Second, we used reversible lesions to test the role of auditory cortex in this task. Third, we used electrophysiological methods to measure activity of single neurons in primary auditory cortex from animals performing the temporal expectation task. These measurements allowed for a characterization of the modulation of neuronal responses by expectation, and an assessment of the relation between the modulation of neural activity and changes in behavior.

\section{Valid temporal expectation improved performance}

We developed an auditory two-alternative choice paradigm, modeled after a related visual paradigm in humans ${ }^{6}$, in which we could study the effect of temporal expectation on performance in rats (Fig. 1). Subjects were rewarded for correctly discriminating the carrier frequency of a frequency-modulated target sound immersed in pure-tone distractors (Fig. 1b). To perform this task the subject had both to detect the target and to discriminate its frequency. The parameters of this task can be manipulated to make either the detection or the discrimination component, or both, arbitrarily difficult.

In the experiments described here, the frequency discrimination component was designed to be easy, so that errors were mainly due to failures of detection. We manipulated detection difficulty by varying target modulation depth (TMD), so that an unmodulated target $(\mathrm{TMD}=0)$ was indistinguishable from a pure tone. On each trial, we presented the target either early (300 or $450 \mathrm{~ms}$ after sound onset) or late (1350 or $1500 \mathrm{~ms}$ after sound onset). We manipulated temporal expectation by presenting trials in blocks of at least 150 trials: expect-early blocks consisted of $85 \%$ trials with early targets and $15 \%$ with late targets, whereas expect-late blocks consisted of $85 \%$ late and $15 \%$ early targets (Fig. 1c). 
Performance depended on the subject's expectation about the moment of occurrence of the target. Valid expectation led to better performance, as assessed by either reaction time (Fig. $2 \mathrm{a}, \mathrm{b})$ or accuracy (Fig. 2c,d) ( $p=0.0078$ paired Wilcoxon signed-rank test). Even for the easiest difficulty tested, where the fraction of correctly detected targets was well above $90 \%$, subjects responded more quickly to early targets when these were expected (Fig. 2a,b).

Reaction time for difficult trials, which is greatly influenced by the correct detection of a target, was even more sensitive to the subjects' expectation (Supplementary Fig. 1, 2). The effect of expectation on accuracy also varied with difficulty.

We focused our analysis on trials with early targets, because in the absence of an early target all late targets can become equally expected (Supplementary Fig. 3). For the easiest stimuli, performance depended only slightly on expectation, but for intermediate difficulties temporal expectation improved performance by as much as $15 \%$ (Fig. 2c,d). These observations demonstrate the influence of temporal expectation not only on the speed of behavioral responses, but also on the perception of target sounds.

\section{Inactivation of auditory cortex decreased performance}

Having established an auditory paradigm for studying the mechanisms of temporal expectation, we next assessed whether the auditory cortex was essential for performing this task. Previous studies have shown that animals can perform various auditory tasks even after bilateral ablation of auditory cortex ${ }^{7,8}$, presumably by means of parallel pathways between subcortical auditory areas and motor regions. To minimize the possibility of cortical reorganization following a permanent lesion ${ }^{9,10}$, we inactivated the auditory cortex reversibly by applying the $\mathrm{GABA}_{\mathrm{A}}$ receptor agonist muscimol bilaterally (Fig. 3a). Using a similar behavioral paradigm, we have previously shown that muscimol inactivation of the auditory cortex impairs auditory performance on a simple auditory discrimination task but has no effect on an olfactory discrimination task ${ }^{11}$.

Muscimol inactivation impaired performance of all animals (Fig. 3b) compared with interleaved saline control sessions ( $p=1.7 \times 10^{-6}$ paired Wilcoxon signed-rank test), although there was considerable animal-to-animal variability in the magnitude of the effect. For the easiest difficulty tested, average reaction times increased significantly from $196 \pm 18 \mathrm{~ms}$ (mean \pm s.e.m.) on saline sessions to $317 \pm 76 \mathrm{~ms}$ on muscimol sessions. In some animals, the effect of inactivation was dramatic, sufficient to reduce performance to chance levels at all tested stimulus difficulties (Supplementary Fig. 5), even though these subjects continued to perform hundreds of trials per session as in control sessions (Supplementary Fig. 6). This reduction in performance supports the hypothesis that the auditory cortex plays an essential role in the task used throughout this study.

\section{Temporal expectation modulated neuronal activity}

We hypothesized that the enhanced performance conferred by valid temporal expectation would be correlated with changes in the neural representation of acoustic stimuli in auditory cortex. To test this hypothesis, we used tetrodes to record responses from single neurons in the primary auditory cortex of three rats performing the temporal expectation task at an intermediate difficulty. To ensure controlled delivery of auditory stimuli to the unrestrained 
rat regardless of the position of its head ${ }^{12}$, sounds were delivered through earphones during recording sessions (see methods).

We focused our initial analysis on the neuronal responses to the tones preceding the target, to understand the effects of target anticipation. To minimize the effects of acoustic context (i.e., the recent history of sounds ${ }^{13,14}$ ), we fixed the frequency of the first three tones for all trials in a given session. We compared responses elicited by these tones between two expectation conditions: expect-early blocks in which the subject expected the target to appear early in the train of sounds, and expect-late blocks in which the target was expected to appear late.

Responses to tones immediately preceding an early target in expect-early blocks were enhanced compared to responses to identical tones in expect-late blocks (Fig. 4). This modulation was observed in neurons with various types of response dynamics: onset, sustained or offset (Fig. 4a and Supplementary Fig. 7). The population of responsive neurons showed an enhancement in evoked activity $\left(p=1.9 \times 10^{-5}\right.$, paired Wilcoxon signedrank test), as indicated by the distribution of modulation indices (Fig. 4b). The low soundevoked firing rates of auditory cortical neurons typically observed in the absence of careful neuron-by-neuron stimulus optimization tends to obscure small changes in firing rate ${ }^{15,16}$. Even so, this enhancement reached significance ( $p<0.05$, Wilcoxon rank-sum test) in almost a third (14/44) of the neurons. Only a single neuron showed a significant change in the opposite direction. A quarter of the responsive neurons (11/44) showed a significant increase of at least $50 \%$ in evoked response; no neuron had a decrease in response of this magnitude. On average, there was no significant change in spontaneous firing rate between conditions for these cells (Supplementary Fig. 8). Stronger responses to stimuli immediately preceding the expected target were also observed in the evoked local field potentials (LFP) (Fig. 4c,d). The observed modulation of evoked responses to sounds occurring around the expected appearance of a target indicates that information about current expectations can enhance the representation of stimuli in early stages of cortical processing.

We then analyzed the effect on the target itself. Surprisingly, a similar analysis of the 26 neurons fulfilling our criteria for responsiveness failed to reveal a systematic effect of temporal expectation (Supplementary Fig. 9). At the end of the following section we provide a possible explanation for this observation.

\section{Modulation was specific to driven activity}

To understand how the modulation by temporal expectation depended on the frequency preference of each neuron, we performed a new set of experiments in which we fixed the frequency of only the first two of the three tones preceding the target and allowed the frequency of the third tone to vary randomly across trials. This modified stimulus allowed for a more accurate estimation of the frequency tuning of each neuron (Supplementary Fig. 10). From the additional recording sessions under these stimulus conditions, 58 cells fulfilled our selection criteria for responsiveness. As above, there was no significant change in spontaneous firing rate between conditions for these cells (Supplementary Fig. 8). 
A priori, the modulation might depend on the relationship between the neuron's preferred frequency and the frequency of either (A) the stimulus; or (B) the targets. To test the first possibility, we analyzed the change in firing with temporal expectation as a function of the difference between the stimulus and preferred frequency. On average, the difference in firing rates between conditions for non-preferred stimuli were smaller than those for the preferred frequency of each cell (Fig. 5), confirming the importance of the relationship between the neuron's preferred frequency and the stimulus frequency. To test the second possibility, we assessed whether the magnitude of the modulation was larger for neurons whose preferred frequency was close to one of the targets. On average, no such relation was found (Supplementary Fig. 11). Furthermore, within the frequency resolution of our stimuli, temporal expectation had no effect on the frequency preference of cells (Supplementary Fig. 12). The dependence of the effect of temporal expectation on the stimulus characteristics, together with the lack of modulation when no stimulus was presented, are consistent with a specific change in the responsiveness of cells representing the stimulus, rather than a nonspecific increase in firing rate.

In light of these findings, we re-analyzed the results of the first set of experiments to assess whether we could account for the lack of modulation of the targets. We hypothesized that this apparent lack of modulation arose from a mismatch between the frequency of the target sounds and the preferred frequency of the recorded cells. We obtained a coarse estimate of the neuron's preferred frequency by quantifying its response to tones preceding late targets. We found that the magnitude of the temporal expectation effect was correlated $(\rho=-0.6$, $p=0.001$, t-test on transformed correlation) with the size of mismatch between the preferred and target frequencies (Supplementary Fig. 13). Thus, neurons did show an increase in evoked response for expected targets, but only when the target matched the neuron's preferred frequency. Because in many cases the target and preferred frequency failed to match, the overall modulation of the target was small and failed to reach significance. This observation may account for the lack of modulation for target sounds described in the previous section.

\section{Responses increased as the time of the target approached}

In addition to the block-by-block comparisons presented above, we tested if there was an increase in evoked response as the expected time of the presentation of a target approached within a trial. In this case, we analyzed the neural responses elicited by the long train of random tones that preceded a late target. This allowed for a rough estimate of the frequency tuning of each cell at various moments within a trial, although on any given trial the specific random sequence of frequencies can exert a strong effect ${ }^{13,14}$. On average, the response to tones at the preferred frequency of each neuron increased as the expected time of the target approached (Fig. 6). Note that this effect is in the direction opposite to the rate-dependent suppression generally observed when presenting a train of sounds of equal relevance ${ }^{12,17,18}$.

\section{Neuronal activity in auditory cortex predicted performance}

The causal role that auditory cortex plays in our task, together with the modulation of activity associated with changes in the subject's cognitive state, suggested a correlation, on a trial-by-trial basis, between neuronal activity and the behavioral improvements resulting 
from temporal expectation. Because of the binary-choice nature of our task, a substantial fraction of rewarded trials may have been correct guesses after missed target detections. As a consequence, an analysis based solely on rewarded vs. not-rewarded trials did not yield significant differences.

To improve our estimates of correct detections, we used reaction times as a surrogate measure of the animal's internal state. As demonstrated in Supplementary Fig. 2, slow behavioral responses were associated with missed detections. We calculated the average neuronal response to stimuli preceding early targets on trials where the subject reacted quickly, and compared these to averages from trials with slow reactions. Indeed, we observed that neuronal responses in auditory cortex to the exact same stimulus were stronger on trials in which the subjects responded quickly (Fig. 7a). This difference in neuronal response was consistent across the population of LFPs $\left(p<10^{-5}\right.$, paired Wilcoxon signedrank test, Fig. $7 \mathrm{~b}$ ) and single cells ( $p=0.018$, paired Wilcoxon signed-rank test, Fig. $7 \mathrm{c}$ ).

An analysis of the correlation between LFP magnitude and reaction time further supports these results. About a fifth of recording sites showed a significant negative correlation between the LFP magnitude and reaction time when including all trials with early targets $(p<0.05$, t-test on transformed correlation). In addition, the average correlation across all sites $\left(\rho_{L F P, R T}=-0.03 \pm 0.01\right.$, mean \pm s.e.m. $)$ was significantly different from zero $(p=0.01$, Wilcoxon signed-rank test), and in the direction indicating faster reaction times on trials with stronger evoked LFP. These observations demonstrate that, even for identical stimuli, the subject's behavioral response is related to neuronal activity in sensory cortex.

\section{DISCUSSION}

We trained rats to perform an auditory task in which expectations about the timing of sounds were manipulated. We quantified the influence of temporal expectation on the subjects' performance of the task, as well as on the activity of single neurons in auditory cortex. In addition, we evaluated the relation between neuronal activity in auditory cortex and behavior by measuring the effects of reversible inactivation, and by correlating evoked activity with performance. We found that: (1) valid temporal expectation leads to improvements in both the speed and accuracy of responses on an auditory detection/discrimination task; (2) the auditory cortex plays a causal role in the performance of this task; (3) neuronal activity in the primary auditory cortex is correlated with the behavioral performance of the subjects on a trial by trial basis; and (4) temporal expectation enhances the neuronal response of cells in the primary auditory cortex. Our results suggest that temporal expectation improves auditory perception by modulating the response properties of single cells at early cortical stages of processing.

\section{Temporal expectations in action and perception}

Processing signals optimally requires exploiting the statistical structure of signals, a principle of central importance in the design of artificial systems ${ }^{19,20}$. In nature, evolution favors the survival of sensory processing systems whose properties are matched to the statistical structure of their environment ${ }^{21}$. A beautiful example is the adaptation of retinal 
ganglion cells to the intensity statistics of visual stimuli so as to make full use of the cells' dynamic range 22 .

Temporal structure on different timescales is an important form of regularity in acoustic signals $^{2}$. For instance, neurons in the auditory cortex respond more strongly to rarely presented stimuli ("oddballs") embedded in a regular sequence ${ }^{3}$, a behavior which can be understood by positing that these neurons have adapted to the recent statistics of their inputs, and that they preferentially represent sounds that are outliers with respect to these recent statistics. In an oddball experiment the statistics are simple, and can be estimated over minutes. On longer time-scales, speech processing requires making predictions based on the statistical structure of language learned over months or years.

In principle, temporal regularities could be exploited to improve performance in at least two distinct ways. First, they might be used to prime the motor system to to respond quickly, leading to a decrease in reaction time but not to an improvement in response accuracy. Alternatively, they could be used to enhance sensory processing at appropriate moments, improving accuracy and also possibly speed. Although several studies have previously demonstrated improved speed consistent with enhanced motor preparedness ${ }^{6,23}$, improved accuracy is typically observed only in perceptually demanding tasks 1,24 .

In our study, regularities in target timing occurred over blocks of either short-delay or longdelay trials. Subjects exploited these regularities to improve both speed (Fig. 2a,b) and accuracy (Fig. 2c,d and Supplementary Fig. 4). Thus we conclude that in our task valid temporal expectation led to improved sound processing, which may or may not also have been associated with increased motor preparedness.

\section{Neuronal mechanisms of temporal expectation}

We hypothesized that the observed improvements in perception due to temporal expectation resulted from changes in the sensory representation of sounds. Because brain areas in the auditory processing stream may be recruited to differing degrees depending on the behavior under study ${ }^{7,8,10}$, we first assessed whether the auditory cortex was essential in this task. Inactivation dramatically reduced performance (Fig. 3a), confirming a role for the auditory cortex. Indeed, the impairment was so large that it precluded evaluating the effect of inactivation on improvements in performance due to temporal expectation. The results of our inactivation experiments, together with potential functional projections into auditory cortex from brain regions that provide expectation or timing signals ${ }^{25-27}$, suggested that the auditory cortex would be a promising area in which to look for neural correlates of temporal expectation.

Single unit tetrode recordings in primary auditory cortex of behaving animals revealed a clear enhancement of activity evoked by stimuli close to a task-relevant moment (Fig. 4). This observation demonstrates that temporal expectation not only influences late stages of processing ${ }^{28,29}$, but can enhance the representation of stimuli as early as primary sensory cortex. These changes in neuronal response are not limited to the representation of rewards ${ }^{30}$, but occur even for non-target sounds that occur near the expected moment of a relevant stimulus. 
Temporal anticipation might be expected to raise the overall level of neuronal activity in a non-specific way. However, no change in spontaneous rate was observed, indicating that the observed changes were specific to driven activity, as often seen with visual attention ${ }^{31,32}$. Furthermore, the difference in firing rate between the expected and unexpected conditions was largest near the neuron's preferred frequency (Fig. 5), indicating that the activity of neurons involved in the representation was preferentially enhanced. Our results are broadly consistent with a multiplicative increase in response above baseline ${ }^{31,32}$, but because of our relatively coarse frequency sampling we cannot assess whether the gain was constant across frequencies. These observations suggest that top-down circuit and synaptic mechanisms underlying temporal expectation may overlap with those underlying other attentional phenomena.

The modulation of activity described in this study should not be interpreted as evidence that the auditory cortex plays a role in keeping the time of relevant events at the scale of seconds, as is observed for example for parietal corte ${ }^{33}$. Instead, we interpret our results as auditory cortex changing its representation of sounds according to timing information it receives from other areas.

In addition to the strong modulation of tones preceding the task-relevant target, our data also suggest an enhancement, albeit subtler, of responses evoked by the target sound itself (Supplementary Fig. 13). The fact that this enhancement of the target was less pronounced than that of the preceding tones was likely a consequence of the details of our experimental design. The small number of unexpected targets of a particular frequency, in addition to the variable duration of the target sound (because sounds stopped once the subject withdrew from the center port), affected the reliability of estimates of responses to target sounds. Nevertheless, responses to both targets and non-targets were consistent with a selective increase in response strength for stimuli close to the expected moment of the target.

\section{Causal role for auditory cortex}

We have described the influence of temporal expectation on behavior as well as on neuronal signals related to sensory stimuli, but these results alone do not link neuronal activity to improvements in performance. Our analysis of correlations between response magnitude and performance (Fig. 7) closes this gap. Our observations indicate that the subject's response is related to neural activity in sensory cortex even for identical stimuli, as has been observed in brain areas causally related to perceptual decisions ${ }^{34}$. This correlation is consistent with the idea that signals in auditory cortex are used by the subject to detect the occurrence of a target, and that changes in these signals can generate improvements in perception.

We have developed an animal model to study the effects of temporal expectation on sounddriven behaviors. This paradigm enabled studying the neuronal mechanisms underlying expectation, beyond what is currently possible in human studies $1,5,35$. Taking advantage of this animal model, we have advanced our understanding of the effects of temporal expectation in the following directions: First, we demonstrated that temporal expectation produces changes in the representation of sensory stimuli, in addition to the observed modulation of motor circuits. Second, we showed that these effects on sensory representation occurred as early as primary sensory cortex, and related this modulation to 
the physiological features of single cells. Last, we provided evidence for a causal link between neuronal activity and behavior, and related improvements in performance to changes in neuronal activity.

\section{METHODS}

\section{Animal subjects}

Animal procedures were approved by the Cold Spring Harbor Laboratory Animal Care and Use Committee and carried out in accordance with National Institutes of Health standards. A total of 14 adult male Long Evans rats (Taconic Farms, Germantown, NY) were used for the experiments: 8 for behavioral analysis (at least 30 sessions per animal), 5 for inactivation experiments ( 10 sessions per animal), and 3 implanted with tetrode microdrives for recordings (two of these included in the behavioral analysis). Rats had free access to food, but water was restricted to behavioral sessions. Free water was provided on days with no experimental sessions. Experiments were conducted in single-walled soundbooths (Industrial Acoustics Company, Bronx, NY).

\section{Behavioral task}

The task consisted of an auditory two-alternative choice paradigm for freely moving rats. Subjects initiated each trial by poking their nose into the center port of a three-port operant chamber. After a silent delay of random duration (250-350 ms, uniformly distributed), a sequence of $100 \mathrm{~ms}$ pure tones separated by $50 \mathrm{~ms}$ was presented. The frequency of the tones, from $5 \mathrm{kHz}$ to $40 \mathrm{kHz}$, was chosen randomly for each time slot and trial, except on recording sessions as described in the main text. Sound intensity was set at $70 \mathrm{~dB}$-SPL. The animal was required to stay in the port until a frequency-modulated target sound (Supplementary Equation 1) was presented in place of one of the distractor tones. The modulation frequency was set to $15 \mathrm{~Hz}$. The carrier frequency of the target indicated to the animal which one of the two side-ports would provide $24 \mu \mathrm{l}$ of water reward on each trial. For a target carrier frequency of $6.5 \mathrm{kHz}$, reward was available only at the left port. For a target at $31 \mathrm{kHz}$, reward was provided at the right port.

Sounds stopped once the subject left the center port. We presented a maximum of 14 sounds $(2.1 \mathrm{sec})$ on a trial. Nothing was presented after this period even if the animal stayed in the center port, but this rarely occurred (less than $2 \%$ of the time). Behavioral analysis included only valid trials in which the animal stayed in the center port until the time of the target onset. Animals were punished with a 4 second time-out (during which nothing was presented) if they withdrew from the center port after the sequence of tones started but before the onset of the target. These events were ignored and the trial was re-started. An equivalent time-out punishment was applied whenever a subject tried to collect reward on the wrong side-port after the presentation of the target. These were considered error trials.

The frequency discrimination component of the task was designed to be very easy for the subjects, and it was used as a way to verify a correct target detection. The challenging component of the task was the detection of the frequency-modulated sound immersed in a sequence of tones, which we could make arbitrarily difficult by reducing its modulation 
depth (TMD). Detection difficulty was randomized for each trial, except on recording sessions where it was set to an intermediate level resulting in an average percentage of correct trials between $70 \%$ and $80 \%$.

Temporal expectation was manipulated in blocks of trials. On an initial block, $85 \%$ of the trials contained the target after 300 or $450 \mathrm{~ms}$ (uniformly distributed) from the first tone onset. In the remaining $15 \%$ of trials, the target was presented after 1350 or $1500 \mathrm{~ms}$. In a following block the ratios were swapped. All four possible target onset times were used in each session, except during recordings where only 450 and $1500 \mathrm{~ms}$ where used. The block type switched automatically every 150 or 200 trials until the session ended. No explicit cue was given to the animal at the end of a block.

\section{Stimulus delivery}

During training and inactivation sessions, auditory stimuli were delivered through generic electromagnetic dynamic speakers calibrated using a pressure-field microphone (Brüel \& Kjær, Norcross, GA) to produce $70 \mathrm{~dB}-\mathrm{SPL}$ in the range 5 to $40 \mathrm{kHz}$ at the position of the subject. During electrophysiological recording sessions, auditory stimuli were delivered through earphones (Etymotic Research, Inc., Elk Grove Village, IL) attached to the head of the animal, and calibrated in a similar way. Waveforms were created in software at a sampling rate of 200,000 samples per second, and delivered to speakers or earphones through a Lynx L22 sound card (Lynx Studio Technology, Inc., Costa Mesa, CA). We applied rise and fall linear envelopes of $2 \mathrm{~ms}$ to all sounds.

\section{Surgery}

Animals were anesthetized with an intraperitoneal injection of a mixture of ketamine (60 $\mathrm{mg} / \mathrm{kg})$ and medetomidine $(0.5 \mathrm{mg} / \mathrm{kg})$. Wounds were infiltrated with lidocaine. For inactivation experiments, plastic wells were implanted to protect bilateral craniotomies over auditory cortex (3 to $6 \mathrm{~mm}$ posterior to Bregma). The dura mater was left intact. For recording experiments, each rat was surgically implanted with a custom-made microdrive in left auditory cortex containing 6 to 14 independently adjustable tetrodes. Electrodes were implanted between 3.5 and $6 \mathrm{~mm}$ posterior to Bregma and $6.5 \mathrm{~mm}$ left from the midline. Animals were allowed to recover for several days before resuming water restriction and starting recording or inactivation sessions. Before recording sessions, earphones were fixed to plastic rings attached to the microdrive during surgery.

\section{Inactivation of auditory cortex}

We used the $\mathrm{GABA}_{\mathrm{A}}$ receptor agonist muscimol (Sigma-Aldrich Corp. St. Louis, MO) to reversibly inactivate auditory cortex bilaterally. After lightly anesthetizing the animal (2\% isoflurane), we placed a gelfoam soaked in $12.5 \mu$ l of either muscimol $(3.44 \mu \mathrm{g} / \mu \mathrm{l})$ or saline $(0.9 \% \mathrm{NaCl})$ on the exposed dura mater on each side. The animal was given 30 minutes to recover from anesthesia before starting the task. Data from at least 5 inactivation and 5 saline-control interleaved sessions were collected for each animal. 


\section{Neural recordings}

Each tetrode consisted of four polyimide-coated nichrome wires (Kanthal Palm Coast, Palm

Coast, FL; wire-diameter $12.7 \mu \mathrm{m}$ ) twisted together and gold-plated to an impedance of 0.3$0.4 \mathrm{M} \Omega$ at $1 \mathrm{kHz}$. Electrical signals passed through unity-gain head-stages (Neuralynx, Inc, Tucson, AZ) connected to a custom-built tetrode microdrive before reaching the acquisition system. Signals were recorded using the NSpike multichannel acquisition system (L. Frank, J. MacArthur). Tetrode depths were adjusted between recording sessions in order to sample an independent population of cells each time. Tetrode locations were confirmed histologically based on electrolytic lesions (Supplementary Fig. 14). While lesions localized to primary auditory cortex, there is the possibility that some of the recorded cells come from non-primary areas since it was not possible to verify the exact location of each recording site. One of the three animals had to be lightly anesthetized (1.5\% isoflurane) before recording sessions to ease the connection of the head-stage and earphones. In this case, a recovery period of 20 minutes was given before the beginning of the task.

\section{Data analysis}

Data were analyzed using in-house software developed in MATLAB (The Mathworks, Natick, MA) and Python (www.python.org). For all analyses, trials after those with unexpected targets were ignored. The first few trials after block switching were not treated any different from subsequent trials. This way, we avoided setting arbitrary thresholds on how many trials constituted the expectation update stage.

Behavioral analysis-Each session included in the behavioral analysis contained interleaved trials of six different difficulties (TMD between $0.15 \%$ and $24 \%$ ) uniformly distributed throughout the session. Reaction time measurements for Fig. 2a,b included only correct trials at the easiest difficulty. At this difficulty level, animals performed above $90 \%$ correct. Reaction time was defined as the time between the onset of a target and the moment when the animal withdrew its nose from the center port. Early targets at both 300 and 450 ms were pooled together when estimating reaction time. Average reaction time for each animal was calculated as the median over trials from all sessions.

The percentage of correct trials for each animal was estimated using trials from all sessions pooled together. Confidence intervals for these estimates were calculated using the MATLAB function binofit which computes the maximum likelihood estimate of the probability of success in a binomial trial based on the number of successes observed in a set of independent trials.

Performance thresholds were estimated by fitting a sigmoidal function (Supplementary Equation 2) to the psychometric data (percent correct $v s$. difficulty) collected for each animal, and finding the TMD that would yield a performance of $P=75 \%$ correct.

Single cell analysis-Spiking activity of a total of 226 single cells was isolated offline by a combination of an automated expectation maximization algorithm (Klustakwik, K.D. Harris) and by manually clustering spike features derived from the sampled waveforms using the MClust software package (A.D. Redish). Only cells with an evoked response of at 
least $3 \mathrm{spk} / \mathrm{sec}$ above spontaneous firing were included in the analysis. This resulted in 102 cells: 44 (out of 127) from sessions where the first three tones had fixed frequencies across trials, and 58 (out of 99) from sessions with only the first two tones fixed.

Average spontaneous firing was evaluated in the $200 \mathrm{~ms}$ window preceding the onset of the first tone. Neural responses were estimated from the most responsive $25 \mathrm{~ms}$ window (given all trials) in the period between the onset of the first tone and the onset of the early target (450 ms later). The magnitude of the response was calculated as the evoked firing rate minus the spontaneous rate.

The modulation index (MI) was defined as:

$$
M I=\frac{R_{\text {expected }}-R_{\text {unexpected }}}{R_{\text {expected }}+R_{\text {unexpected }}}
$$

where $R$ is the evoked response, measured as spikes per second or magnitude of LFP (Fig. $4 \mathrm{~b}, \mathrm{~d})$. This quantity is bounded between -1 and 1 for positive evoked changes in activity, which was the case in our measurements. The statistical significance of the modulation of activity for single cells was evaluated with a Wilcoxon rank-sum test on the spike counts (over the response window) from each expectation condition. For the population, we used a paired Wilcoxon signed-rank test on the average response for each condition to evaluate significance. Because there were fewer trials available for each frequency when estimating the tuning of a cell, response curves (to generate Fig. 5) were calculated from a variable length window around the maximal response. The window was formed by all contiguous $25 \mathrm{~ms}$ bins that showed an evoked response of at least $70 \%$ of the maximum. For this figure, neuronal activity is presented without subtracting spontaneous firing in order to test for nonspecific modulation. Individual tuning curves were aligned according to the preferred frequency of each cell and normalized before averaging.

To evaluate the changes in response over time across the population of cells (Fig. 6), we first estimated the best frequency for each unit by pooling together all responses to tones between the early and the late targets. We then evaluated the response to the best frequency tone at each time slot, and normalized it to the response to the earliest tone in this range (the fourth tone in the sequence, presented $1050 \mathrm{~ms}$ before the late target). Responses were estimated using the same $25 \mathrm{~ms}$ time window with respect to each tone onset. Only cells with responses above spontaneous firing for each time-slot were included $(\mathrm{N}=20)$. Statistical significance at each time slot was evaluated with a paired Wilcoxon signed-rank test with respect to the fourth tone.

Local field potentials-The magnitude of the evoked local field potential (LFP) was calculated as the root mean square (RMS) power of the signal in the period 310-360 ms after the onset of the stimulus (Supplementary Equation 3). This period corresponds to the onset response to the tone preceding the time of early targets. For comparisons of evoked LFP magnitudes between conditions (Fig. 4c,d and Fig. 7), trials were first averaged separately for each condition. Changes in evoked LFP magnitudes were quantified by a 
modulation index between conditions, as described above. Statistical significance for individual sites on a given comparison was evaluated using permutation tests.

Correlation between evoked responses and behavior-To evaluate the relation between neuronal activity and behavioral performance, we compared the neuronal activity of trials with early targets grouped according to reaction time. On each individual behavioral session, we sorted trials according to reaction time and selected two groups: one made of the $20 \%$ trials with fastest behavioral responses, and a second one made of the $20 \%$ trials with slowest behavioral responses. We estimated average response magnitudes for both LFP and single cells, as described in previous sections, and calculated a modulation index in each case to evaluate the differences.

Statistical significance of the correlation between LFP magnitude and reaction time was calculated using the function corrcoef from MATLAB, which transforms the correlation values to create a t-statistic in order to estimate the probability of getting a correlation as large as the observed value by random chance.

\section{Supplementary Material}

Refer to Web version on PubMed Central for supplementary material.

\section{ACKNOWLEDGEMENTS}

This research was supported by a postdoctoral fellowship from the Swartz Foundation, and by grants from the Swartz Foundation, the NIH and the Marie Robertson Fund. We thank G. H. Otazu, A. E. Baker, J. M. Abolafia and B. Burbach for assistance with preliminary studies.

\section{References}

1. Nobre A, Correa A, Coull J. The hazards of time. Current Opinion in Neurobiology. 2007; 17(4): 465-470. [PubMed: 17709239]

2. Winkler I, Denham SL, Nelken I. Modeling the auditory scene: predictive regularity representations and perceptual objects. Trends in Cognitive Sciences. 2009; 13(12):532-540. [PubMed: 19828357]

3. Ulanovsky N, Las L, Nelken I. Processing of low-probability sounds by cortical neurons. Nat Neurosci. 2003; 6(4):391-398. [PubMed: 12652303]

4. Fritz J, Shamma S, Elhilali M, Klein D. Rapid task-related plasticity of spectrotemporal receptive fields in primary auditory cortex. Nat Neurosci. 2003; 6(11):1216-1223. [PubMed: 14583754]

5. Lange K, Rosler F, Roder B. Early processing stages are modulated when auditory stimuli are presented at an attended moment in time: an event-related potential study. Psychophysiology. 2003; 40(5):806-817. [PubMed: 14696734]

6. Nobre AC. Orienting attention to instants in time. Neuropsychologia. 2001; 39(12):1317-1328. [PubMed: 11566314]

7. Ohl FW, Wetzel W, Wagner T, Rech A, Scheich H. Bilateral ablation of auditory cortex in mongolian gerbil affects discrimination of frequency modulated tones but not of pure tones. Learning \& Memory. 1999; 6(4):347-362. [PubMed: 10509706]

8. Syka J, Rybalko N, Mazelová J, Druga R. Gap detection threshold in the rat before and after auditory cortex ablation. Hearing Research. 2002; 172(1-2):151-159. [PubMed: 12361878]

9. Newsome W, Paré E. A selective impairment of motion perception following lesions of the middle temporal visual area (MT). J. Neurosci. 1988; 8(6):2201-2211. [PubMed: 3385495]

10. Talwar SK, Musial PG, Gerstein GL. Role of mammalian auditory cortex in the perception of elementary sound properties. J Neurophysiol. 2001; 85(6):2350-2358. [PubMed: 11387381] 
11. Tai, L.; Zador, A. Available from Nature Precedings. 2008. Neural mechanisms of selective auditory attention in rats (Dissertation). <http://dx.doi.org/10.1038/npre.2008.2355.1>

12. Otazu GH, Tai L, Yang Y, Zador AM. Engaging in an auditory task suppresses responses in auditory cortex. Nat Neurosci. 2009; 12(5):646-654. [PubMed: 19363491]

13. Bar-Yosef O, Rotman Y, Nelken I. Responses of neurons in cat primary auditory cortex to bird chirps: Effects of temporal and spectral context. J. Neurosci. 2002; 22(19):8619-8632. [PubMed: 12351736]

14. Asari H, Zador AM. Long-Lasting context dependence constrains neural encoding models in rodent auditory cortex. J Neurophysiol. 2009; 102(5):2638-2656. [PubMed: 19675288]

15. Hromádka T, DeWeese MR, Zador AM. Sparse representation of sounds in the unanesthetized auditory cortex. PLoS Biol. 2008; 6(1):e16. [PubMed: 18232737]

16. Wang X, Lu T, Snider RK, Liang L. Sustained firing in auditory cortex evoked by preferred stimuli. Nature. 2005; 435(7040):341-346. [PubMed: 15902257]

17. Brosch M, Schreiner CE. Time course of forward masking tuning curves in cat primary auditory cortex. J Neurophysiol. 1997; 77(2):923-943. [PubMed: 9065859]

18. Wehr M, Zador AM. Synaptic mechanisms of forward suppression in rat auditory cortex. Neuron. 2005; 47(3):437-445. [PubMed: 16055066]

19. Shannon CE. A mathematical theory of communication. Bell System Technical Journal. 1948; 27:379-423. 623-656.

20. Haykin, S. Adaptive Filter Theory. 4th edition. Prentice Hall; 2001.

21. Rieke, F.; Warland, D.; de Ruyter van Steveninck, R.; Bialek, W. Spikes: Exploring the Neural Code. The MIT Press; 1996.

22. Smirnakis SM, Berry MJ, Warland DK, Bialek W, Meister M. Adaptation of retinal processing to image contrast and spatial scale. Nature. 1997; 386(6620):69-73. [PubMed: 9052781]

23. Niemi P, Näätänen R. Foreperiod and simple reaction time. Psychological Bulletin. 1981; 89(1): 133-162.

24. Correa Á, Lupiáñez J, Tudela P. Attentional preparation based on temporal expectancy modulates processing at the perceptual level. Psychonomic Bulletin and Review. 2005; 12(2):328-334. [PubMed: 16082814]

25. Kilgard MP, Merzenich MM. Cortical map reorganization enabled by nucleus basalis activity. Science. 1998; 279(5357):1714-1718. [PubMed: 9497289]

26. Tremblay L, Schultz W. Relative reward preference in primate orbitofrontal cortex. Nature. 1999; 398(6729):704-708. [PubMed: 10227292]

27. Rao SM, Mayer AR, Harrington DL. The evolution of brain activation during temporal processing. Nature Neuroscience. 2001; 4:317-323. [PubMed: 11224550]

28. Ghose GM, Maunsell JHR. Attentional modulation in visual cortex depends on task timing. Nature. 2002; 419(6907):616-620. [PubMed: 12374979]

29. Anderson B, Sheinberg DL. Effects of temporal context and temporal expectancy on neural activity in inferior temporal cortex. Neuropsychologia. 2008; 46(4):947-957. [PubMed: 18206961]

30. Shuler MG, Bear MF. Reward timing in the primary visual cortex. Science. 2006; 311(5767): 1606-1609. [PubMed: 16543459]

31. Treue S, Trujillo JCM. Feature-based attention influences motion processing gain in macaque visual cortex. Nature. 1999; 399(6736):575-579. [PubMed: 10376597]

32. McAdams CJ, Maunsell JH. Effects of attention on Orientation-Tuning functions of single neurons in macaque cortical area v4. J. Neurosci. 1999; 19(1):431-441. [PubMed: 9870971]

33. Janssen P, Shadlen MN. A representation of the hazard rate of elapsed time in macaque area LIP. Nat Neurosci. 2005; 8(2):234-241. [PubMed: 15657597]

34. Britten KH, Newsome WT, Shadlen MN, Celebrini S, Movshon JA. A relationship between behavioral choice and the visual responses of neurons in macaque MT. Visual Neuroscience. 1996; 13(1):87-100. [PubMed: 8730992] 
35. Correa Á, Lupiáñez J, Madrid E, Tudela P. Temporal attention enhances early visual processing: a review and new evidence from event-related potentials. Brain Res. 2006; 1076(1):116-128. [PubMed: 16516173]

36. Uchida N, Mainen ZF. Speed and accuracy of olfactory discrimination in the rat. Nat Neurosci. 2003; 6(11):1224-1229. [PubMed: 14566341]

37. Paxinos, G.; Watson, C. The rat brain in stereotaxic coordinates. 5th edition. Academic Press; 2005. 

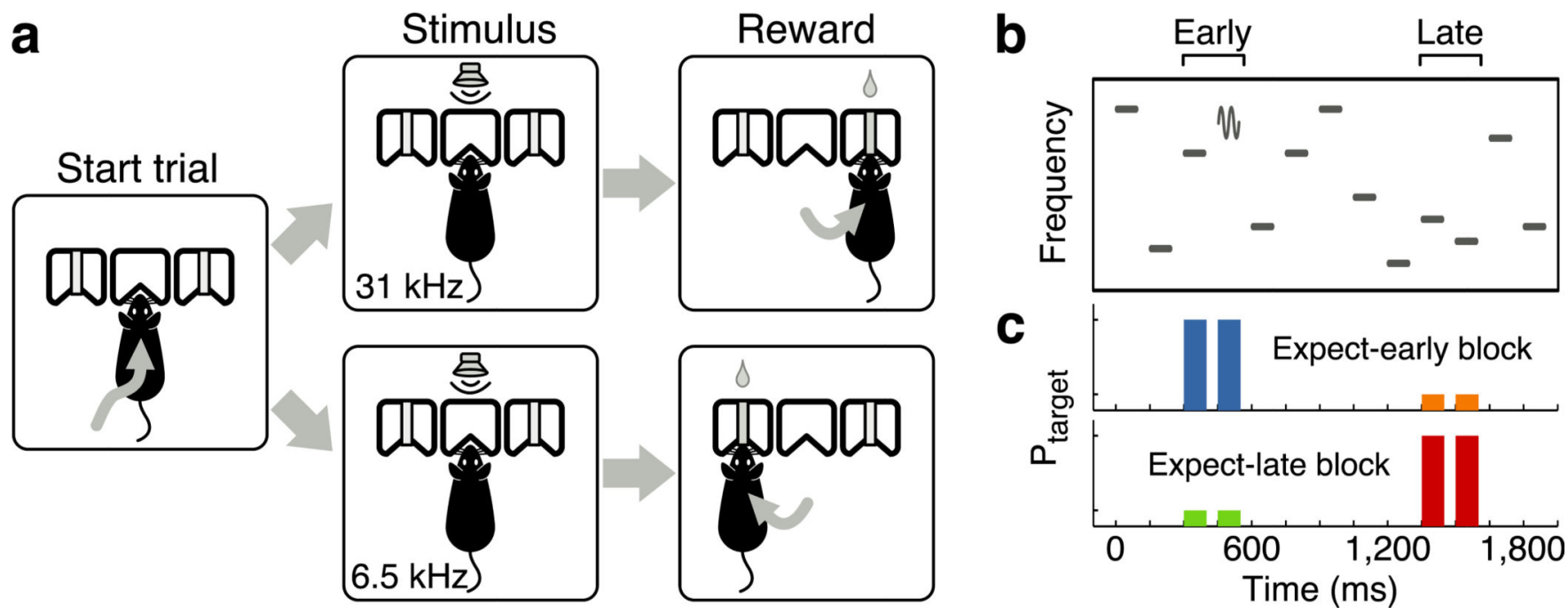

Figure 1. Task and manipulation of temporal expectation

(a) Subjects initiated each trial by poking into the center port of the operant chamber ${ }^{12,36}$. After a variable (250-350 ms) silent period, a stimulus consisting of a frequency-modulated target immersed in a train of pure tone distractors was presented. Subjects were required to stay in the port until the target was presented. The center frequency of the target (either 6.5 $\mathrm{kHz}$ or $31 \mathrm{kHz}$ ) indicated the side-port where water reward would be delivered on each trial (left or right, respectively).

(b) The stimulus consisted of a sequence of 100ms pure tones (ranging from 5 to $40 \mathrm{kHz}$ ) separated by $50 \mathrm{~ms}$, which was presented for as long as the animal stayed in the port. The frequency-modulated target was presented in place of one of the tones in each trial.

(c) Temporal expectation was manipulated by changing the ratio of trials with early or late targets within each block of 150-200 trials. 

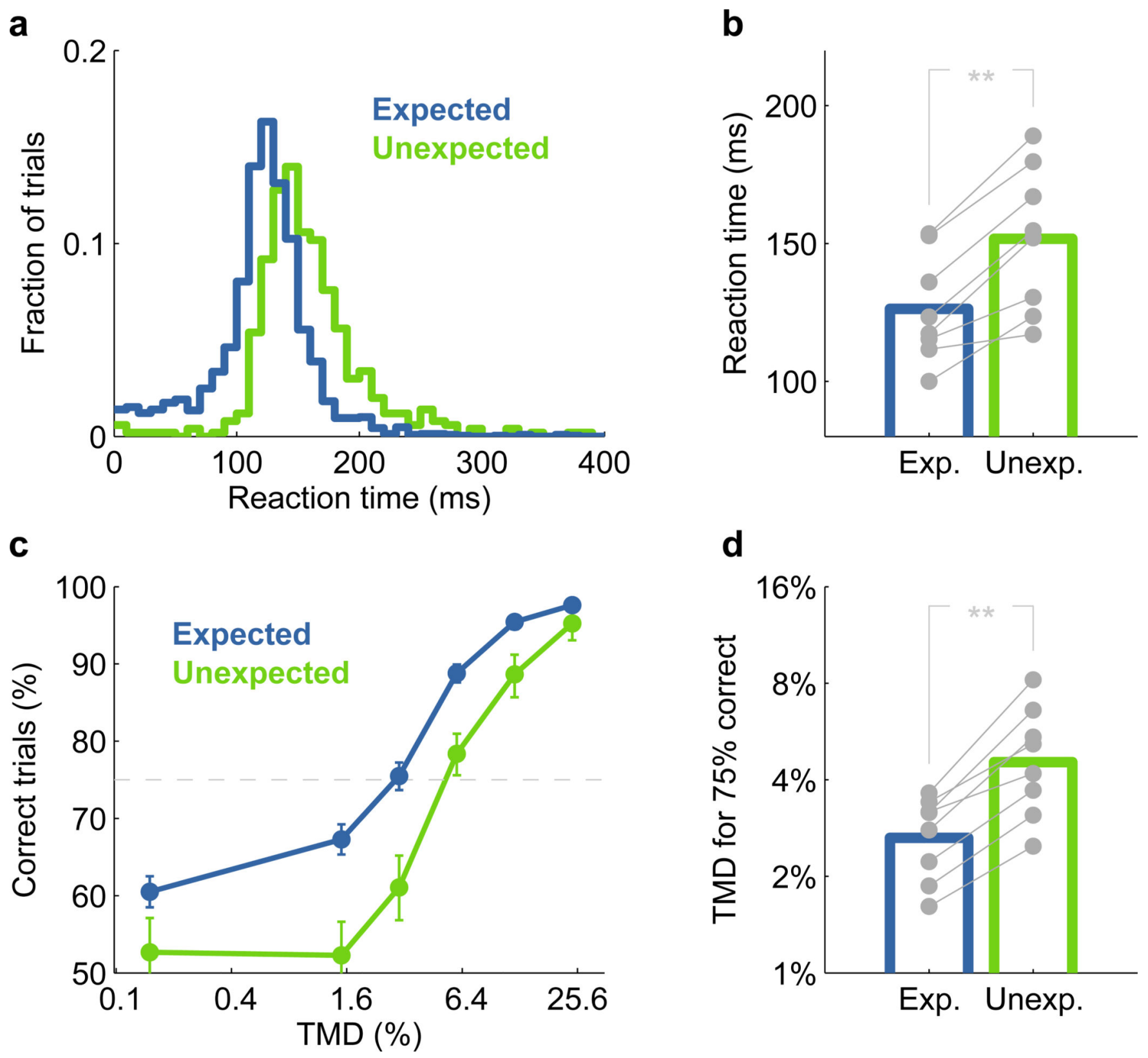

Figure 2. Valid temporal expectation improved performance

(a) Behavioral responses were faster on trials with expected targets. Example of the reaction time distribution from one animal at the easiest difficulty tested (TMD 25\%) for early targets that were expected (blue) or unexpected (green). Reaction time was defined as the time between the onset of a target and the moment when the animal left the center port.

(b) Median reaction time for each animal (gray dots) on the easiest difficulty tested, and average across all 8 subjects for early targets that were expected (blue) or unexpected (green). Stars represent statistical significance $(* * p<0.01)$.

(c) Behavioral responses were more accurate on trials with expected targets. Example for one animal of the percentage of correct trials as a function of difficulty, varied here by the modulation depth of the target (TMD). Error bars correspond to the $95 \%$ confidence 
intervals on estimates. The dashed line corresponds to $75 \%$ performance used for calculating thresholds in $\mathbf{d}$.

(d) Modulation depth needed to achieve 75\% correct trials for each subject (gray dots), and average across all 8 subjects (colored bars). Stars represent statistical significance (** $p<0.01)$. 

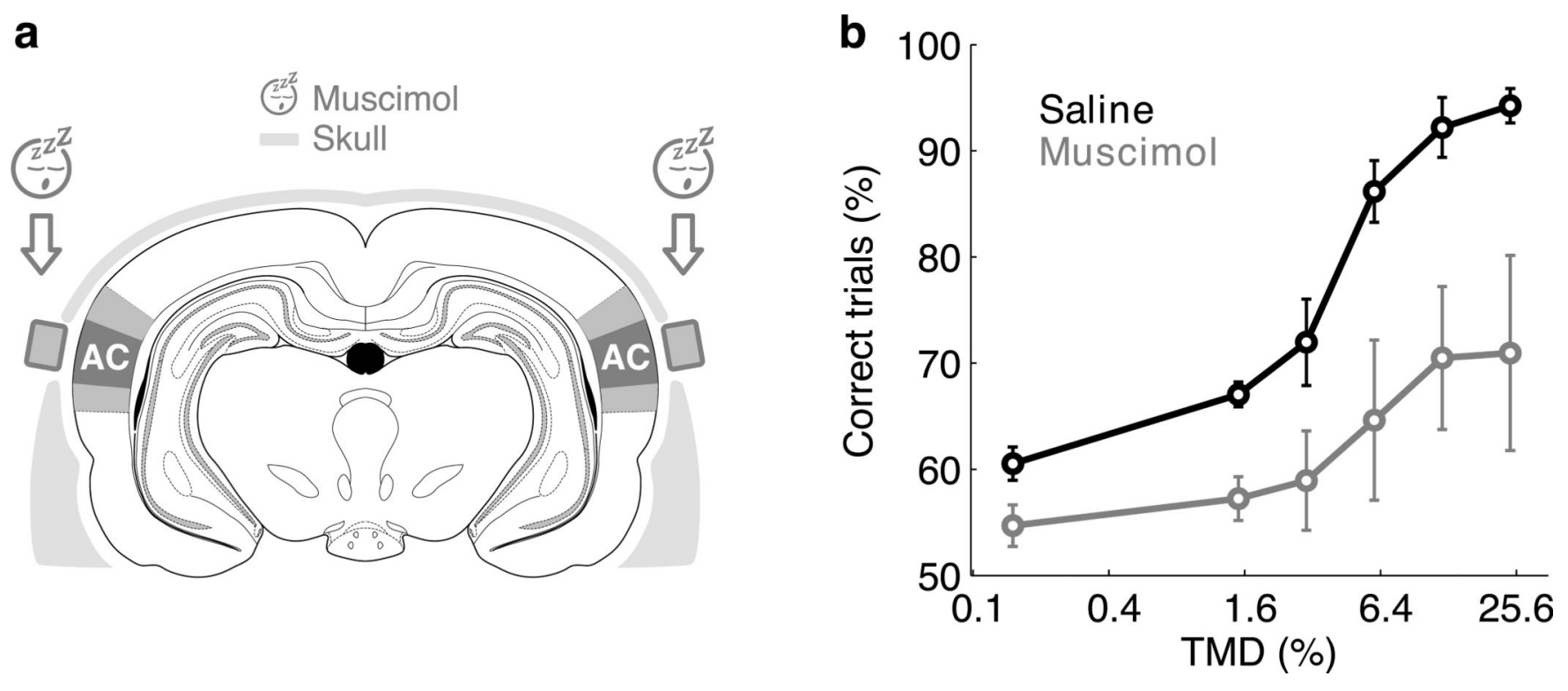

Figure 3. Inactivation of auditory cortex decreased performance

(a) Bilateral reversible inactivation of auditory cortex (AC) was performed by applying the $\mathrm{GABA}_{\mathrm{A}}$ receptor agonist muscimol on the surface of the exposed dura mater. Craniotomies were protected by implanted wells. Darker and lighter colored regions indicate primary and secondary auditory cortices respectively ${ }^{37}$.

(b) Performance on expected early targets as a function of difficulty on interleaved inactivation (gray) and control (black) sessions. The plot shows the average over 5 animals. Error bars indicate s.e.m. across animals. 

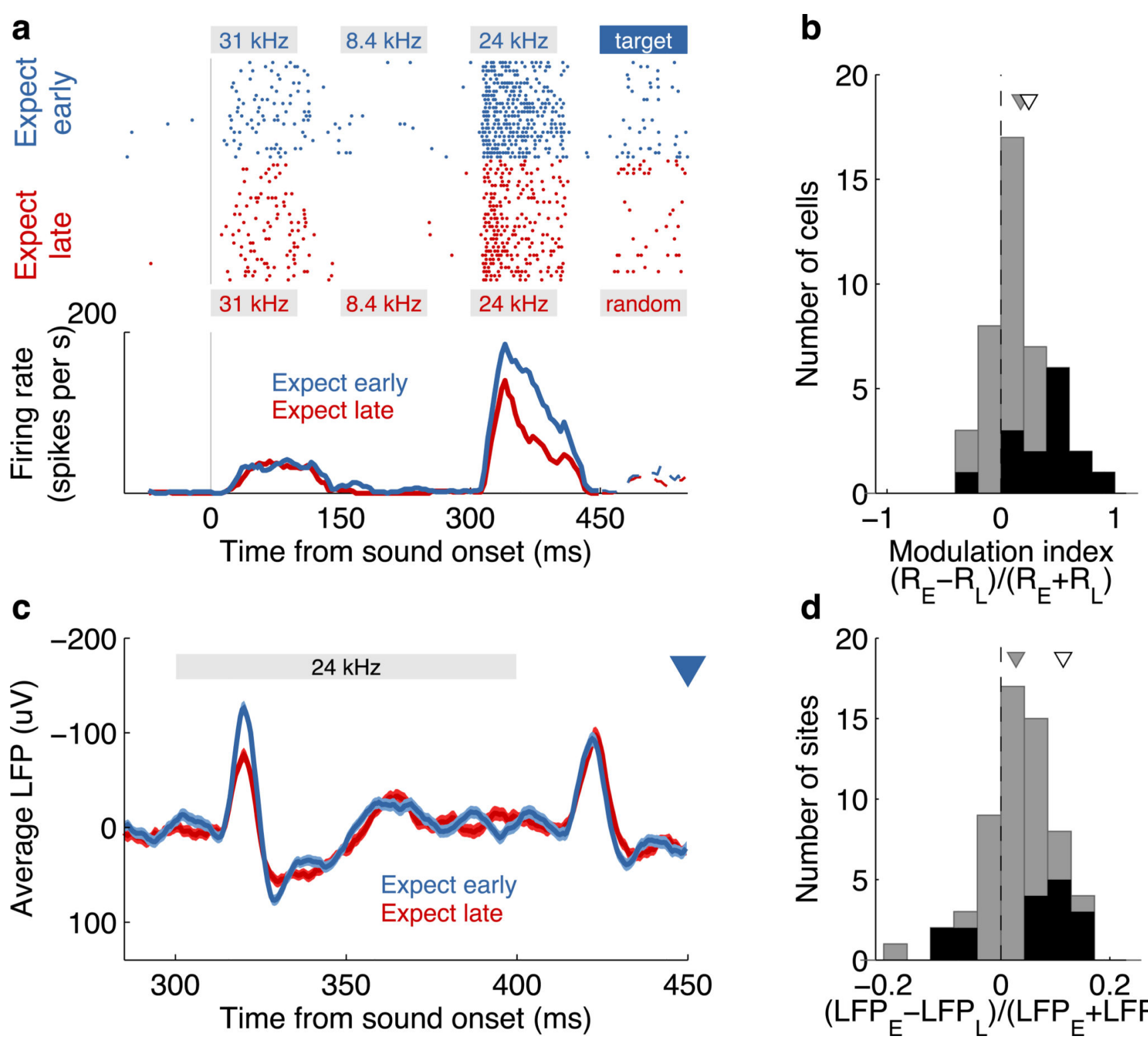

Figure 4. Temporal expectation modulated neuronal activity in auditory cortex

(a) Responses of a single neuron to the same sequence of tones under two temporal expectation conditions: expecting an early (blue) or a late (red) target. Expected early targets appeared after $450 \mathrm{~ms}$, whereas expected late targets (not visible here) were presented after $1500 \mathrm{~ms}$. Trials are aligned to the onset of the first tone (gray vertical line) for the spike raster (top) and the peristimulus time histogram for each condition (bottom). The session included more than two blocks of trials, but all expect-early or expect-late blocks are grouped together here for illustration purposes. The frequency and duration of each tone is indicated by the gray boxes. The largest difference in evoked activity is seen for the tone immediately preceding the early target. The stimuli presented after $450 \mathrm{~ms}$ are not the same on each trial; average responses in this case are shown as dashed lines.

(b) Modulation index of 44 responsive cells recorded during sessions where all tones preceding the early targets had fixed frequencies. A positive modulation index indicates a 
stronger response on expect-early trials. Cells with statistically significant modulation ( $p<0.05$ Wilcoxon rank-sum test) are shown in black. The gray triangle indicates the mean of the modulation index. The white triangle shows the modulation index for the example in a.

(c) Evoked local field potential (mean \pm s.e.m.) at one recording site. The onset of the early target is indicated by the blue triangle.

(d) Modulation of local field potentials. Colors as in $\mathbf{b}$. 
a

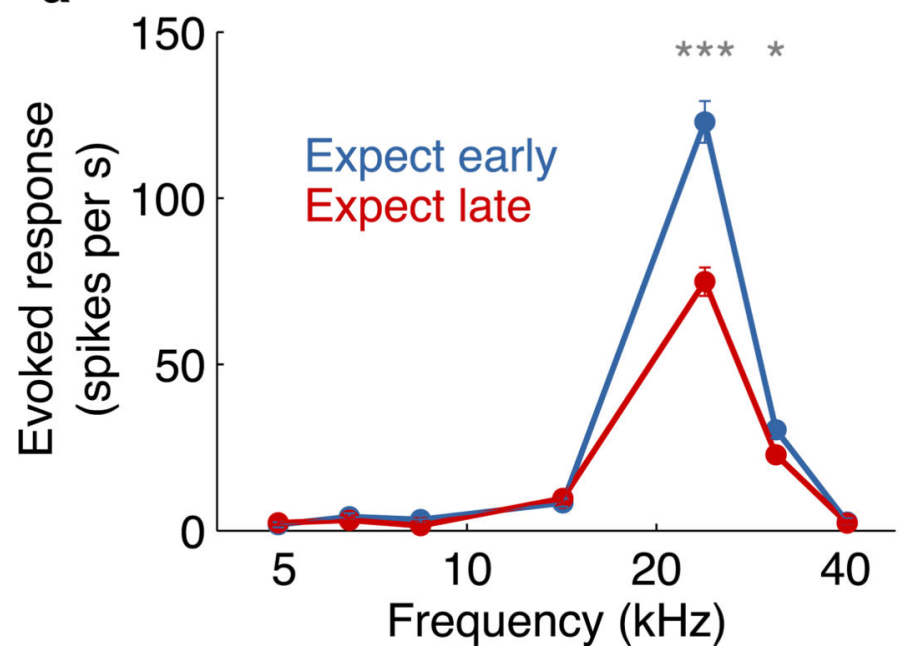

b

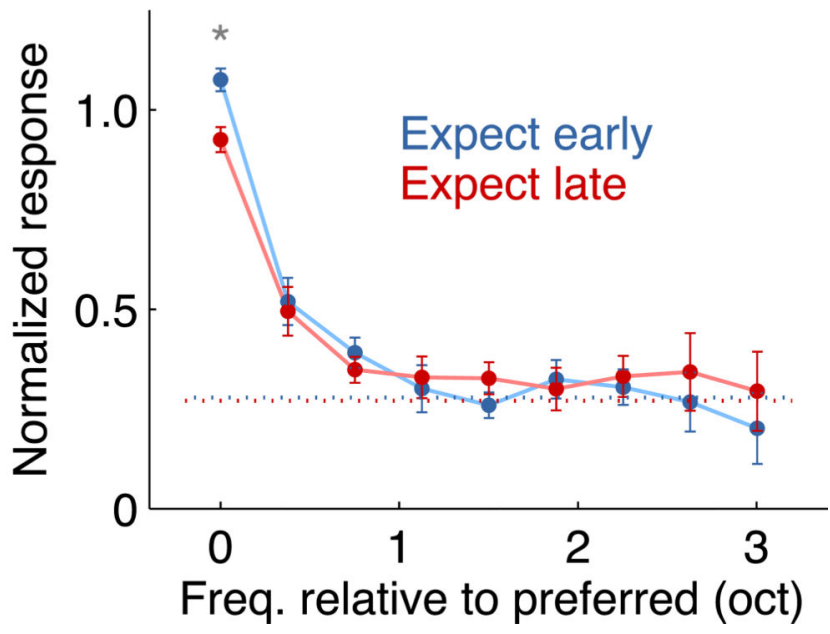

Figure 5. Modulation of neuronal activity was specific to driven activity

(a) Frequency tuning of a single cell, estimated from responses to the third tone in each expectation condition (see Supplementary Fig. 10). Each marker represents the mean \pm s.e.m. firing rate for each tone frequency. Significance levels are indicated by stars (* $p<0.05, * * * p<0.001$, Wilcoxon rank-sum test).

(b) Average frequency tuning of 58 cells recorded with a third tone of random frequency. Individual tuning curves were aligned according to the preferred frequency (PF) of each cell and normalized before averaging. Each marker indicates the mean \pm s.e.m. across neurons. Significance levels are indicated by stars $(* p<0.05$, paired Wilcoxon signed-rank test). 
a

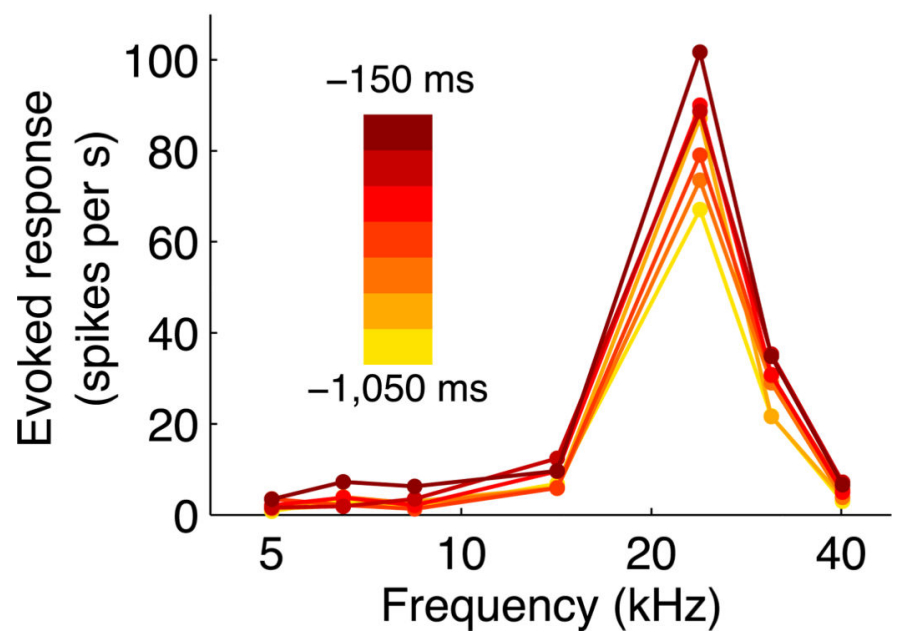

b

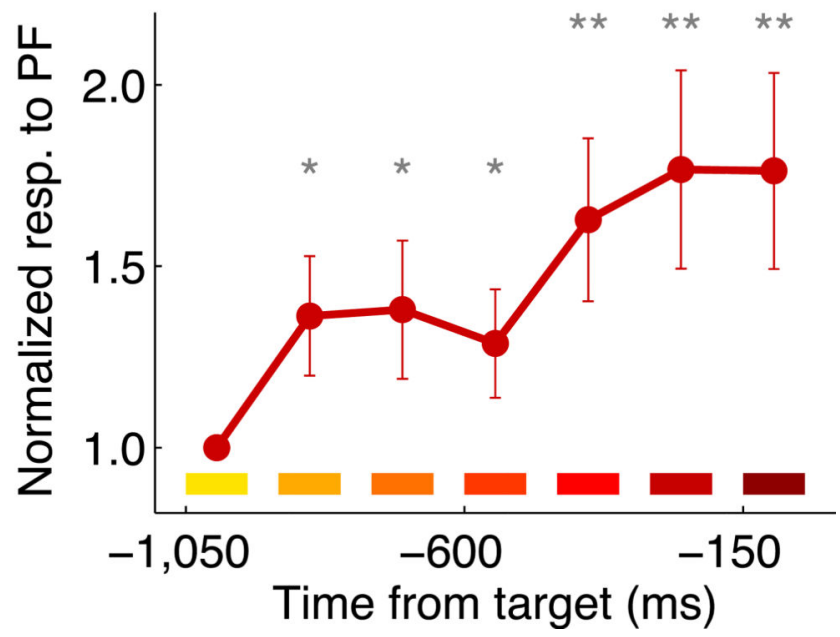

Figure 6. Neuronal response increased as the expected moment of the target approached (a) Frequency tuning of cell in Fig. 5a, as the time of the late target approaches. For each time-slot preceding the late target, the estimated tuning curve is plotted in a different color. The color-bar shows the time of each time-slot with respect to the target onset.

(b) Neuronal response to each cell's preferred frequency (PF) as the time of the target approaches. Responses were normalized with respect to the response to the fourth tone $(-1050 \mathrm{~ms}$ from late target onset). Each point corresponds to the median across cells, with error bars proportional to the median absolute deviation. Only responses to the preferred frequency for each cell are presented, and only cells with responses above spontaneous firing for each time-slot were included $(\mathrm{N}=20)$. The significance level of the difference with respect to the response to the fourth tone is indicated at each time-slot by stars $(* p<0.05$, ** $p<0.01$, paired Wilcoxon signed-rank test). 


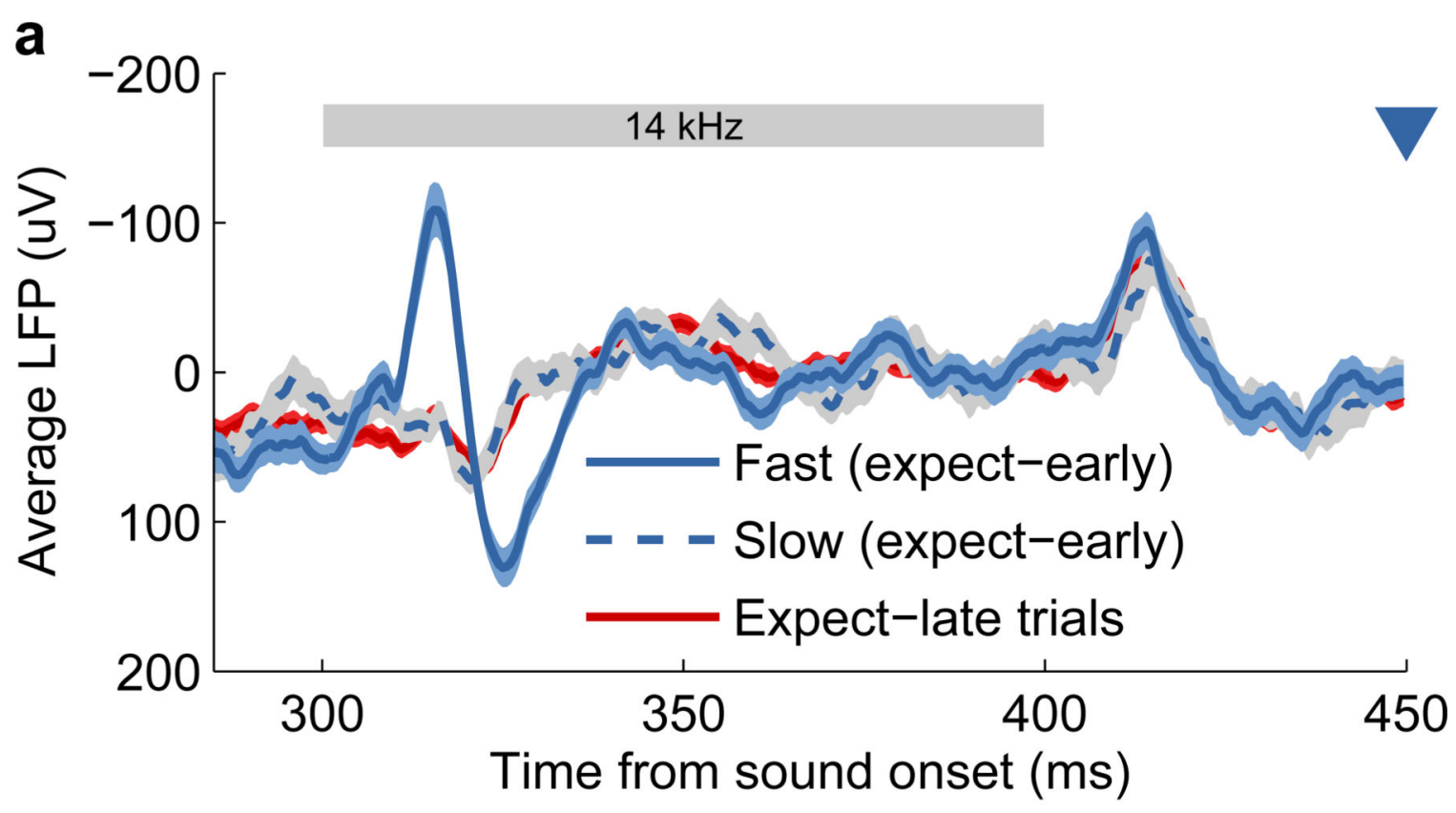

b
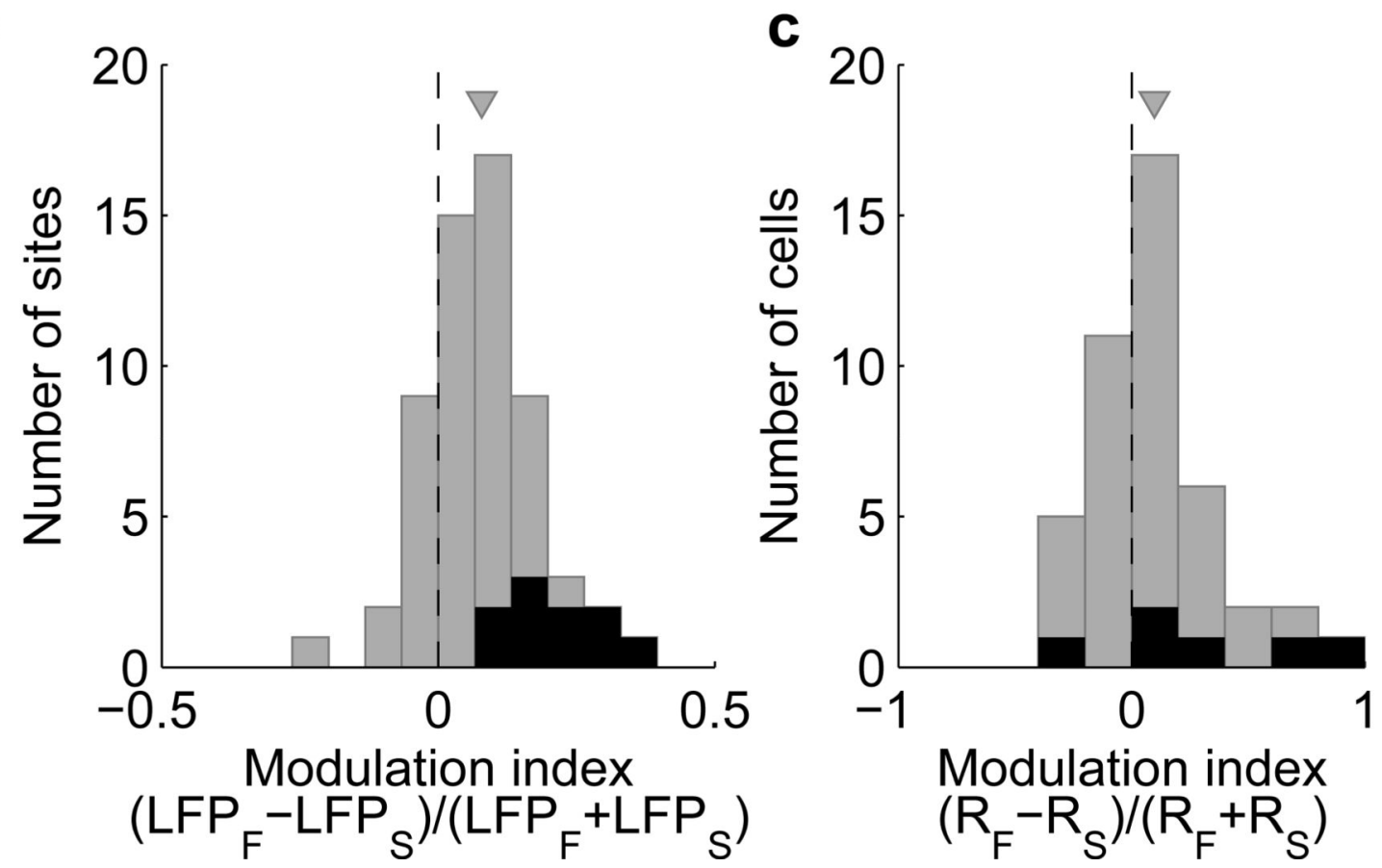

Figure 7. Neuronal activity in auditory cortex was correlated to behavioral performance (a) Average evoked LFP from a single electrode for trials with expected early targets grouped according to reaction time. Averages are taken over those trials with the $20 \%$ fastest (blue solid), or the $20 \%$ slowest (blue dashed) reaction times. Evoked LFP for trials with late targets is shown in red for comparison. The light-colored bands surrounding each trace indicate the s.e.m. across trials. The stimulus (third tone with fixed frequency across trials) is indicated by the gray bar, and the onset time of an early target is represented by the blue triangle. 
(b) Evoked LFPs were larger on trials with faster behavioral responses. Difference in evoked LFP magnitude between trials with the fastest and slowest behavioral responses. The difference is quantified by a modulation index between the average response on fast $\left(\mathrm{LFP}_{F}\right)$ and slow $\left(\mathrm{LFP}_{S}\right)$ trials for each recording site $(\mathrm{N}=59)$. Sites with a significant difference $(p<0.05$, permutation test) are shown in black. The gray triangle indicates the mean modulation index.

(c) Evoked spiking activity was larger on trials with faster behavioral responses. Difference in evoked activity on single cells between trials with the fastest and slowest behavioral responses. The difference is quantified by a modulation index between the average response on fast (RF) and slow (RS) trials for each cell $(\mathrm{N}=44)$. Cells with a significant difference $(p<0.05$, Wilcoxon rank-sum test) are shown in black. The gray triangle indicates the mean modulation index. Only sessions where the third tone had the same frequency on all trials were included in this analysis. 